\title{
Enabling Self-Diagnosis of Automation Devices through Industrial Analytics
}

\author{
Carlos Paiz Gatica and Alexander Boschmann \\ Weidmüller Interface GmbH \& Co. KG, Klingenbergstraße 16, 32758 Detmold, Germany \\ \{carlos.paizgatica; alexander.boschmann\} @weidmueller.com
}

\begin{abstract}
This paper shows how automation components can be enhanced with self-monitoring capabilities, which are more effective than traditional rule-based methods, by using Industrial Analytics approaches. Two application examples are presented to show how this approach allows the realization of a predictive maintenance strategy, while drastically reducing the realization effort. Furthermore, the benefits of a flexible architecture combining edge- and cloud-computing for the realization of such monitoring system are discussed.
\end{abstract}

Keywords: Industrial Analytics, Predictive Maintenance, Machine Learning, Edge Computing, Feature Engineering, Self-Monitoring.

\section{Motivation and Application Areas}

The realization of predictive maintenance strategies in nowadays production facilities is a complex endeavor. Given the rather heterogeneous landscape of typical production facilities, where machines at different stages of their life cycle and from different vendors are combined for a single production line, this situation is even more challenging. In many cases, unplanned downtime is caused by components lacking monitoring capabilities (e.g., dedicated monitoring sensors), which force plant operators to increase the maintenance efforts to guaranty a steady operation. One promising way to drastically reduce the costs of maintenance is the use of Industrial Analytics approaches. Here, the use of data from the production system combined with machine learning methods and domain knowledge leads to the realization of monitoring systems able to automatically detect changes in the behavior of a machine or a component during operation or to predict undesirable situations.

There is a need for flexibility in the realization of Industrial Analytics functions to address the long range of industry applications. For machinery applications, data sets are generated from control systems operating in real time. The applied algorithms need to operate with short reaction times to avoid critical failures or to decrease quality problems resulting on the production of scrap. In these kind of applications, the required sensor data is rather small and the sensor signals are highly correlated to each other. Therefore, an implementation of industrial analytics functions using edge devices alone or in combination with cloud computing brings many advantages, such as short reaction times and decreasing network traffic. 
This paper shows the use of Industrial Analytics as means of enabling a condition based- or even a predictive maintenance strategy for simple automation components lacking dedicated monitoring resources. It is shown in section 2 how a flexible architecture combining edge and cloud computation enables the realization of such monitoring system. The process to develop an Industrial Analytics solution is then explained in section 3. Two practical use cases are then presented in section 4, disclosing the potential of this approach to reduce maintenance costs while increasing its effectiveness.

\section{Development process of Industrial Analytics solutions}

Industrial analytics functions are typically composed of different tasks, as shown in Figure . The figure shows the typical workflow of an industrial analytics application, where data from the different devices are first consolidated in a single data source (data storage). The next step is to pre-process the data as preparation for the learning process (preprocessing). In this step, relevant features are extracted from the raw data signals, involving the combination of statistical methods with domain-knowledge to select meaningful features.

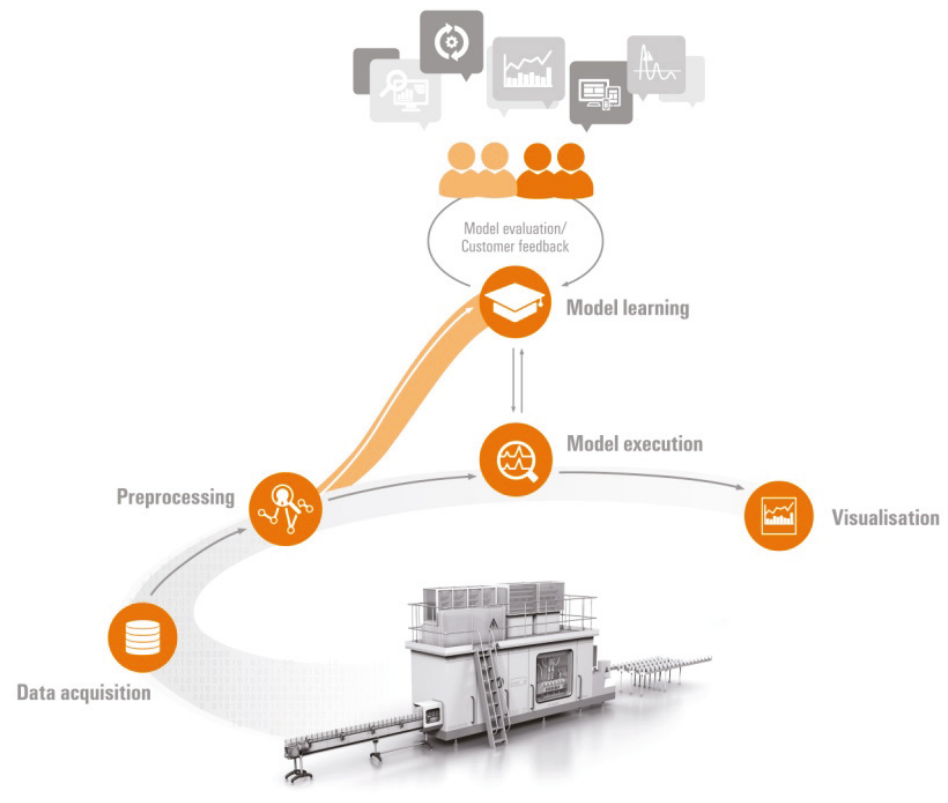

Figure 1: Typical workflow of an industrial analytics system.

The next step is the selection, training and tuning of machine learning algorithms to derive a model from the selected features (model learning). Again, the combination of analytics expertise and domain knowledge is key to develop an efficient model. Once developed, the model can be used at runtime to monitor the machine or process (model execution). To be useful the results need to be properly visualized (visualization). The kind of visualization should be selected according to the role of the person who shall 
use this information, e.g., the machine operator, the maintenance manager, etc. The integration of an industrial analytics function in an automation system can be done at different levels, for instance at the machine, or using a cloud platform. These possibilities are explored in the next section.

\section{A flexible automation system architecture for Industrial Analytics}

In a typical automation system, the continuous stream of heterogeneous data created by machines, actuators and sensors can be used as input for industrial analytic applications such as predictive maintenance. As more and more smart components from the Internet of Things (IoT) domain enter the production facilities, this flood of data will grow dramatically and will become increasingly difficult to manage utilizing a centralized Cloud-based data collection and processing approach. The concept of Edge Computing has recently been proposed to overcome this limitation by providing a distributed computing model where data is processed at the "edge" of a network, i.e., near field devices [SD2016, GJFVR2016].

The core benefit of this approach is to allow for low latency by computing the data where it is created without incurring network latencies, which is essential for real-time condition monitoring applications. Another benefit is scalability: while a traditional centralized approach will no longer be feasible with an increasing number of communicating devices, Edge Computing provides a linear scalability and is needed as augmentation to reduce pressure on network infrastructure. Furthermore, storage and operation cost can be reduced by processing time-sensitive data locally and significantly reducing raw data before being sent to the Cloud. This technique can also be used to preserve privacy by ensuring that sensitive data is pre-processed on-premise so that only privacycomplaint data is transferred to the Cloud. Following the steps from data acquisition to analytics processing and to the visualization of meaningful machine information, various processing steps at different system components are involved. Figure 1 illustrates an example of a flexible automation system architecture implementing Industrial Analytics at Edge-, on-premise- and Cloud levels.

Raw data are acquired by Remote Terminal Units (RTUs) from machines, and process-relevant actuators and sensors over a fieldbus, e.g. PROFIBUS, depicted by green bus connections. An initial pre-processing stage such as filtering can be implemented on these devices. The signals are then collected by a Programmable Logic Controller (PLC) and used to control the system. Additional process-independent components like smart temperature-, vibration- or pressure sensors are typically connected to an Industrial IoT (IIoT) gateway via Bluetooth, WiFi, Ethernet or the emerging 5G [PLZW2015]. These components play an important role in the process of retrofitting and enabling Industrial Analytics services on older machines. Monitoring systems for important control parts that usually don't offer data interfaces by design (i.e. electromechanical relays or solenoid valves) can ideally be connected to an IIoT Gateway. We present two practical use cases for these systems in the following section of this paper. 


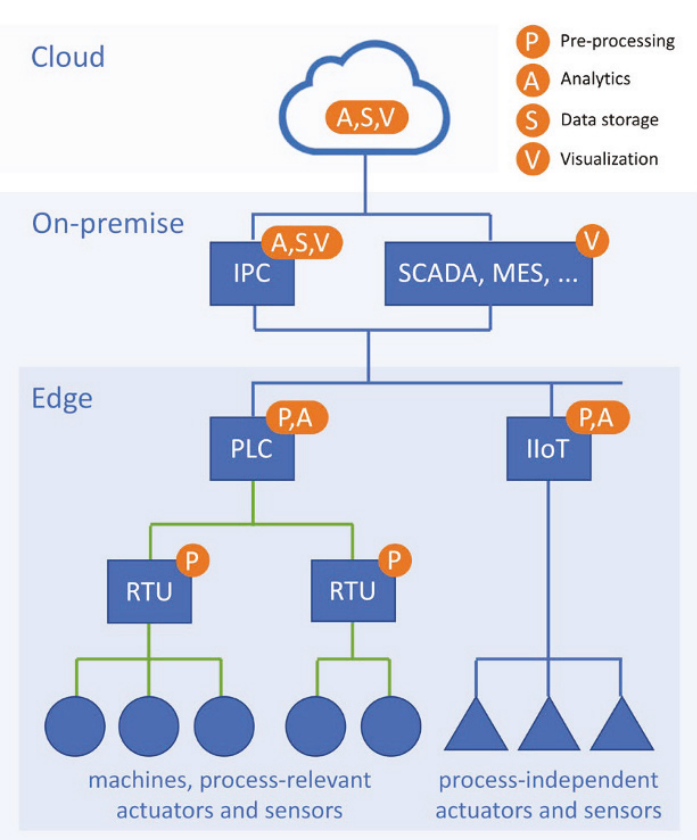

Figure 2: A flexible automation system architecture for Industrial Analytics.

Low latency Edge Analytics functions can be implemented in both, modern PLCs and IIoT gateways. While the PLC can only monitor the devices connected to it, the IIoT gateway typically can access the PLC data in addition to the process-independent component data to generate a larger machine learning model. If necessary, the data density can be further decreased at the Edge level. In addition to data storage and visualization, more complex analytics functions over multiple machines or devices can be performed on-premise by an Industrial PC (IPC) or in the Cloud at the cost of higher latency and increased network traffic. Rich and detailed visualization functions are offered by the Supervisory Control and Data Acquisition (SCADA) or Manufacturing Execution System (MES).

\section{Use Cases}

In this section two use cases are presented, which show the benefits of enabling simple automation devices with self-monitoring capabilities: Monitoring of electromechanical relays and solenoid valves.

\section{Monitoring of Electromechanical Relays}

Electromechanical relays are electrically operated switches that use an electromagnet to mechanically operate a switch to control a circuit by a separate low-power signal. 
They are widely used in industrial areas such as plant construction, mechanical engineering or shipbuilding for switching inductive loads, e.g. for controlling solenoid valves.

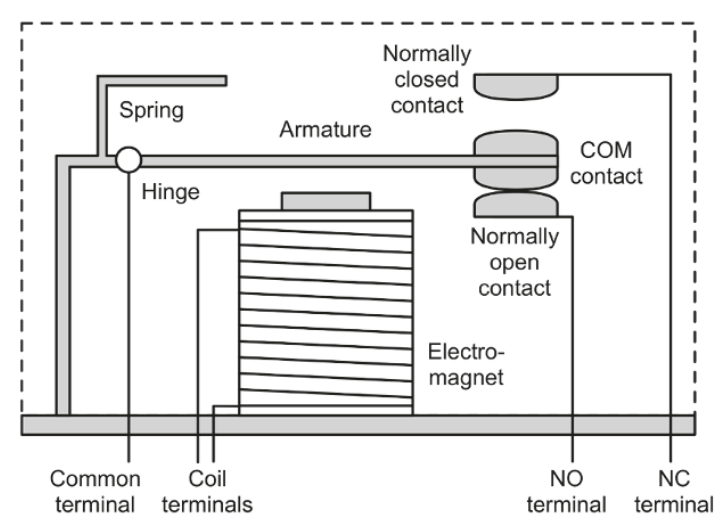

Figure 3: Cross section of a typical electromechanical relay

A simple electromechanical relay consists of an electromagnetic coil, a movable armature and contacts. The armature is attached with a spring so that under normal working conditions it comes back to its original position. If the coil is supplied by the source, a magnetic field causes to attract the armature towards the electromagnet so that the normally open contact (NO) and common terminal contact (COM) connect. This state is shown in Figure 3. When the coil is not supplied by the source, there is no magnetic flux production and the spring draws the armature to its original position so that the normally closed contact (NC) and COM connect. The heavy load on the relay contacts $\mathrm{NC}$ and NO that repeatedly occurs while switching inductive DC loads causes premature failure of the relay. Depending on the application, downtime, equipment damage or personal injury can result from component failure. For this reason, it is important to replace damaged relays in time.

In this use case, electromechanical relays were tested for inductive load over their lifetime to develop Industrial Analytics methods for failure detection. In the experimental setup, relays were tested by switching on and off repeatedly under a high DC load. An inductive load was connected to the contact side of the relays, causing an arc between the opening contact surfaces at the moment of switch-off and damaging the relay contacts. This process was repeated until failure of the relay.

A combination of features based on the electric current flow through the relay coil in combination with a Kullback-Leibler divergence-based classifier [KL1951] has been found which allows for a prediction of imminent failure and predictive maintenance. In this study, only features that can be directly measured in the relay without additional sensors were considered. Figure 3 shows an example plot of the classification output. 


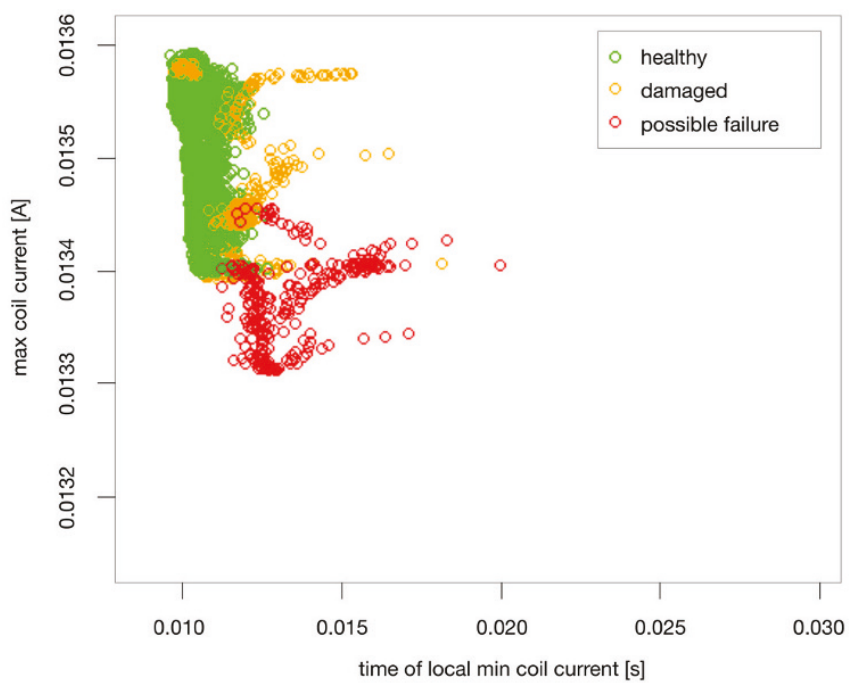

Figure 4: Example classification output of the relay condition monitoring method

Here, the relays were classified into three categories: healthy (green), damaged (orange) and possible failure (red). With the method presented in this paper it is possible to detect an imminent failure due to welding of the relay contacts with high accuracy. In this case, a condition monitoring system can trigger a warning and initiate a predictive maintenance measure before actual damage has occurred. The time remaining in a concrete use case scenario to respond to the imminent failure depends heavily on the switching frequency of the relay being monitored. Based on our experiments, the method presented here allows enough reaction time for applications having high switching frequencies (10 operations per second) or low switching frequencies (1 operation per hour). For this kind of applications, analytics

\section{Monitoring of solenoid valves}

Solenoid valves are among the most important control units in today's industry. Especially in the process industry, solenoid valves play an important role because they control the media flow of gases and liquids.

When a current is applied to the magnet winding, the movable magnet armature is attracted, thus releasing the valve plug from the valve seat (see Figure 5). A medium can flow. When switching off the current, the return spring ensures the lowering of the magnet armature and thus the closure of the valve seat by the valve plug. Mechanical loads on the moving parts and the permanent flow of media cause signs of wear inside the solenoid valve. Also, the continuous use under difficult operating conditions, such as high temperatures and vibrating environments, can cause additional wear. Since solenoid valves are often used in safety-critical applications, malfunctions can have catastrophic economic consequences and, above all, put in danger human lives. Not only is wear within a solenoid valve a safety hazard, errors in the signal line (e.g., wire break, short circuit) to the solenoid valve can also cause failures and thus pose a high risk. 


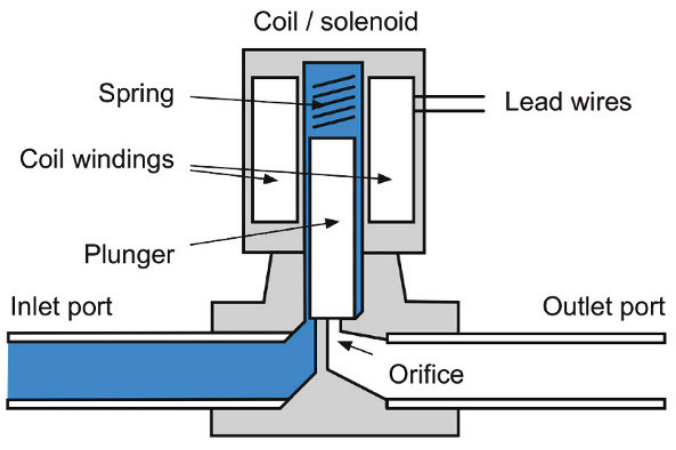

Figure 5: Schematic of a solenoid valve

To prevent premature wear due to wear, the valve or drain in the solenoid valve and the signal line to the valve must be monitored. Four error classes dominate the reports [NRC1987]:

- Foreign matter in the valve $(16 \%)$

- Burnt coil / short circuit (15\%)

- Worn or defective valve parts $(11 \%)$

- Open circuit in coil ( $9 \%)$

When monitoring solenoid valves, there are two different approaches. The first approach is a rule based approach. During operation, the load current is monitored by means of an electronic component. If the current falls below or exceeds the set limits, the block sends a signal to the controller.

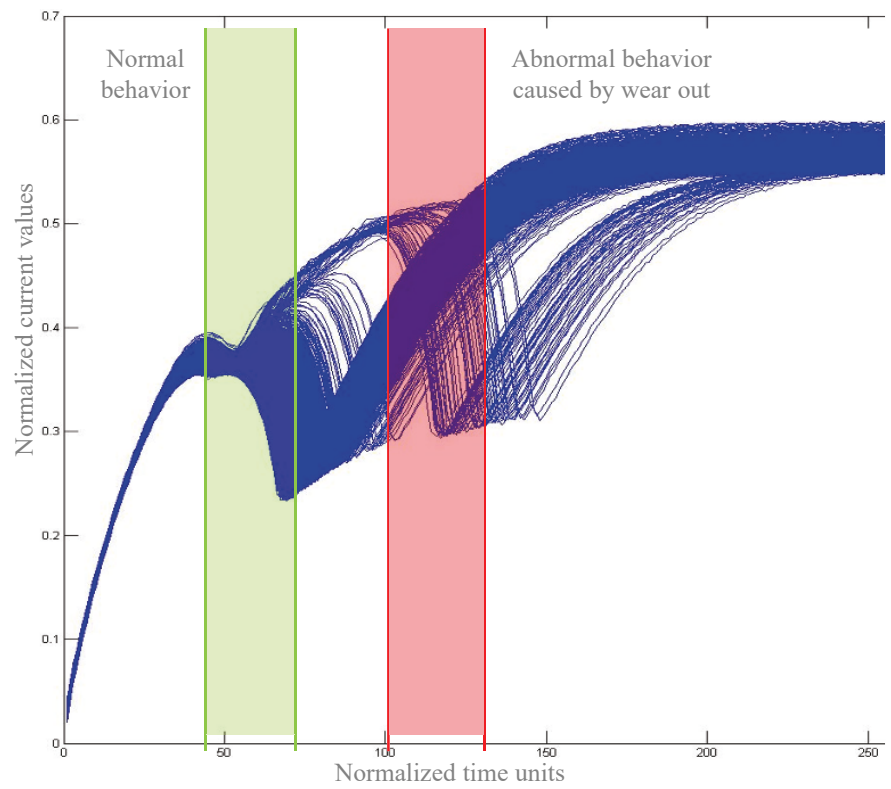

Figure 6: A significant shift in the curves indicates signs of wear on the valve mechanism 
With this method, events such as wire breakage, short circuit or overvoltage and undervoltage can be detected and reported. However, changes in the dynamics of the system inside the defined boundaries are not detected.

The second approach pursues the goal of early detection of valve failure. Here, the current waveforms of switching cycles are recorded and compared (see Figure 6). This approach enables device- and application-specific monitoring, because the reference model is created or parameterized during operation. Deviations to a certain extent may indicate a near defect and thus initiate the timely replacement of the valve (see Figure 6 ). As in the previous case, the realization of this monitoring strategy does not require the use of dedicated sensors, because features extracted from already existing signals are used. This enables the realization of such strategy also for low cost applications.

\section{Summary and Conclusions}

This paper has shown the use of Industrial Analytics as means of enabling a predictive maintenance strategy. It is shown how a flexible architecture for the realization of datadriven monitoring enables the realization of such monitoring system also for simple automation devices. This is demonstrated by two practical use cases, disclosing the potential of this approach to reduce maintenance costs while increasing its effectiveness.

\section{References}

1. [SD2016] W. Shi and S. Dustdar, "The Promise of Edge Computing," in Computer, vol. 49, no. 5, pp. 78-81, May 2016.

2. [GJFVR2016] D. Georgakopoulos, P. P. Jayaraman, M. Fazia, M. Villari and R. Ranjan, "Internet of Things and Edge Cloud Computing Roadmap for Manufacturing," in IEEE Cloud Computing, vol. 3, no. 4, pp. 66-73, July-Aug. 2016.

3. [PLZW2015] M. Peng, Y. Li, Z. Zhao and C. Wang, "System architecture and key technologies for 5G heterogeneous cloud radio access networks," in IEEE Network, vol. 29, no. 2, pp. 6-14, March-April 2015.

4. [KL1951] Kullback, S.; Leibler, R. A. On Information and Sufficiency. Ann. Math. Statist. 22 (1951), no. 1, 79--86.

5. [NRC1987] Aging and service wear of solenoid-operated valves used in safety systems of nuclear power plants: Volume 1, Operating experience and failure identification (Nuclear Regulatory Commission, Washington, DC, USA, 1987) 
Open Access This chapter is licensed under the terms of the Creative Commons Attribution 4.0 International License (http://creativecommons.org/licenses/by/4.0/), which permits use, sharing, adaptation, distribution and reproduction in any medium or format, as long as you give appropriate credit to the original author(s) and the source, provide a link to the Creative Commons licence and indicate if changes were made

The images or other third party material in this chapter are included in the chapter's Creative Commons licence, unless indicated otherwise in a credit line to the material. If material is not included in the chapter's Creative Commons licence and your intended use is not permitted by statutory regulation or exceeds the permitted use, you will need to obtain permission directly from the copyright holder.

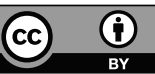

